\title{
Spin-resolved photoemission from Xe(111) by circularly polarized light: experiment and theory
}

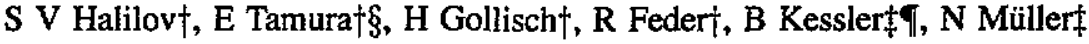 \\ and U Heinzmannị \\ † Theoretische Festkörperphysik, Universität Duisburg, D-W-4100 Duisburg, Federal Republic \\ of Germany \\ ¥ Fakultät für Physik, Universität Bielefeld, D-W -4800 Bielefeld, Federal Republic of \\ Germany and Fritz-Haber-Institut der Max-Planck-Gesellschaft, Faradayweg 4-6, D-W-1000 \\ Berlin, Federal Republic of Germany
}

Received 16 December 1992, in final form 2 March 1993

\begin{abstract}
Spin-resolved photoemission spectra from solid $\mathrm{Xe}(111)$ have been measured using circularly polarized synchrotron radiation with photon energies ranging from $11-18 \mathrm{eV}$. Corresponding calculations by a fully relativistic one-step-model layer KKR formalism produce fairly good agreement with the experimental data. The spectra can be interpreted in terms of direct interband transitions in the real part of a complex-potential band structure. This provides detailed information on the quasi-particle band structure including real and imaginary self-energy corrections to the valence and conduction bands. Observed 'opposite-spin features', which are absent in the calculated spectra, are tentatively interpreted as due to electron-hole scattering processes.
\end{abstract}

\section{Introduction}

Because of spin-orbit coupling, spin-resolved photoemission spectroscopy-obviously a method of choice for magnetic systems-is also firmly established as a powerful tool for studying in detail the electronic structure of non-magnetic crystals and their surfaces (see, for example, reviews by Wöhlecke and Borstel (1984), Feder (1985), Heinzmann and Schönhense (1985), Meier (1985), Kirschner (1985) and Heinzmann (1990), and representative original articles by Eyers et al (1984), Kessler et al (1987), Tamura et al (1987, 1989), Schneider et al (1989), Stoppmanns et al (1991) and Tamura and Feder (1991a,b) and references therein). Maximal information can be obtained if experimental data are analysed and interpreted with the aid of realistic numerical calculations of spin-resolved photoemission spectra-using a fully relativistic one-step-model formalism-and of the underlying relativistic quasi-particle band structure for initial and final states. The real and imaginary self-energy corrections, which are at present hardly available from first principles, can be determined by comparing calculated spectra with their experimental counterparts.

Spin-resolved photoemission studies have so far mostly focused on metals and semiconductors. The first experimental investigation on an insulator was performed by Kessler et al (1987) on crystalline xenon. In the present work, we revisit Xe(111) more 
thoroughly. We present new measured spin-resolved spectra for photon energies ranging between $11-18 \mathrm{eV}$ and compare them with their calculated counterparts. Thereby we obtain detailed information on the underlying quasi-particle band structure. In particular, we find that the measured spectra can be understood in terms of direct bulk interband transitions only if an imaginary self-energy part is assumed in calculating the unoccupied states.

In sections 2 and 3 we outline the experimental set-up and the theoretical model assumptions, respectively. The measured and calculated photoemission spectra, as well as the quasi-particle band structure, are presented and discussed in section 4 .

\section{Experiment}

The experiment was performed at the $6.5 \mathrm{~m}$ nomal-incidence monochromator beamline for circularly polarized light at BESSY in Berlin (Schäfers et al 1986) with a polarization degree of $(90 \pm 3) \%$. The photoemission apparatus has been described before (Eyers et al 1984, Kessler et al 1987). The Xe (111) crystals were grown epitaxially on clean $\mathrm{Pt}(111)$ surfaces cooled by liquid He to about $40 \mathrm{~K}$. No charging was observed with a typical sample thickness of approximately 10-15 Xe layers. A photoemission geometry with normal incidence of the light and normal electron emission was used. The overall energy resolution (monochromator plus $180^{\circ}$ spherical-field electron-energy analyser) was better than $0.2 \mathrm{eV}$ (FWHM). The spinpolarization component, $P$, normal to the sample surface (along the direction of the incident light) was measured by Mott scattering at $100 \mathrm{keV}$.

The spin-dependent data are represented by totally spin-polarized partial intensities $I_{+}, L_{-}$, which are related to the spin polarization $P$ and the total (spin-summed) intensity $I_{0}$ by $I_{ \pm}=\frac{1}{2} I_{0}(1 \pm P)$. Here $I_{+}$is the partial intensity totally polarized parallel to the spin of the photons, i.e. $P>0$ means that photon spin and electron spin are parallel.

Due to an acceleration voltage of $5 \mathrm{eV}$ between the sample and the spectrometer the acceptance of the photoemission setup depends slightly on the kinetic energy of the electrons $E_{\text {kin }}$ : while electrons with $E_{\text {kin }}=1 \mathrm{eV}$ are accepted within a cone of $\pm 4^{\circ}$, this cone is narrowed to up to $\pm 3^{\circ}$ for larger electron energies. As well as this deviation from nomal emission a misalignment uncertainty of $0.5^{\circ}$ of the sample has to be taken into account.

\section{Theory}

We employ a fully relativistic one-step-model layer KKR photoemission formalism, which was developed and presented earlier (Ackermann and Feder (1985a, b), Ackermann (1985), Tamura et al (1987), Tamura and Feder (1991a,b), and references therein). We note in particular that in our present formalism the initial state is treated by a Green function method, which, in contrast to an earlier Bloch-wave treatment, allows an a priori incorporation of the hole lifetime. Simultaneously, we calculated the fully relativistic bulk band structure for occupied and unoccupied states.

The effective complex potential, in the muffin-tin approximation, was constructed as follows. We first performed a self-consistent LMTO calculation, which is scalar relativistic throughout and includes spin-orbit coupling in the last cycle, for bulk Xe using the von Barth-Hedin (1972) exchange correlation approximation. For the lattice constant we used a value of $6.13 \AA$ (Kittel 1971). Calculations for the larger value of $6.197 \AA$ 
(Wyckoff 1982) produce almost the same band structure. The resulting real potential was then employed inside the atomic spheres. The uniform part of the LMTO potential is certainly inadequate for photoemission purposes: it is real, i.e. it fails to account for lifetime effects, and it does not depend on energy, which in particular implies far too small an optical gap (between valence band maximum and conduction band minimum). Part of the latter deficiency may also be a shortcoming of the local density approximation. We therefore employed inner potential parameters based on experimental information and to be verified retrospectively by the agreement between calculated and measured photoemission spectra. We thus chose the real part of the inner potential as $6.85 \mathrm{eV}$ for the occupied bands, such as to place the valence-band maximum $9.8 \mathrm{eV}$ below the vacuum level (Kessier et al 1987), and as $2.70 \mathrm{eV}$ for the unoccupied bands corresponding to a self-energy correction of $4.15 \mathrm{eV}$. This is larger than the correction values of $3.15 \mathrm{eV}$ (Timmer and Borstel, as quoted by Kessler et al 1987) and $2.7 \mathrm{eV}$ (Bacalis et al 1988) obtained in approximate first-principles calculations. The discrepancy is explained as follows. Firstly, the latter calculations neglect spin-orbit coupling. Comparing our fully relativistic LMTO results with scalar relativistic ones, we find that spin-orbit coupling reduces the LMTO optical gap by $0.52 \mathrm{eV}$, which entails a corresponding enhancement of the required self-energy correction. Secondly, the $2.7 \mathrm{eV}$ correction to scalar relativistic LDA bands (Bacalis et al 1988) is still $0.9 \mathrm{eV}$ less than the $3.6 \mathrm{eV}$ required to produce the experimental optical gap value. Augmenting the $3.6 \mathrm{eV}$ by the spin-orbit contribution of $0.52 \mathrm{eV}$ in fact gives very good agreement with our photoemission-derived self-energy correction of $4.15 \mathrm{eV}$. The imaginary part of the inner potential was taken as $-0.025 \mathrm{eV}$ for both the occupied and the unoccupied states. The geometry for the photoemission calculation was taken as a simple truncation of the bulk.

\section{Results and discussion}

We now proceed to our measured and calculated results for normal emission from Xe (111) for normally incident circularly polarized photons of various energies.

In figure 1 we present measured and calculated partial intensity spectra $I_{ \pm}$for photon energies from 11-13.5 eV. Let us first focus on the panel for $12.5 \mathrm{eV}$. Experiment and theory are seen to agree very well with regard to existence, energetic position and spin preference of the three prominent features labelled A, B and C. Inspection of the occupied part of the band structure in figure 2 reveals that these features originate from initial states of double-group symmetry types $\Lambda_{4+5}, \Lambda_{6}$ and $\Lambda_{6}$, respectively. Their spin polarization sign is thus as expected from relativistic dipole selection rules with final states of $\Lambda_{6}$ symmetry. We recall that only these fully symmetric states can couple to the vacuum, i.e. reach the detector. The vertical lines in figure 2 indicate that peak $C$ can be interpreted in terms of a direct interband transition in the usual real band structure. For the smaller peaks A and B, however, the final-state energy lies in a gap. But this gap is bridged in the real part of the band structure calculated for the complex upper-state potential including an imaginary selfenergy correction part $V_{\mathrm{i} 2}=-0.025 \mathrm{eV}$. A direct transition interpretation, to quasi-particle final states, can therefore be maintained.

More information is obtained by considering the evolution of the spectra (figure 1) over a range of photon energies. The theoretical results can consistently be interpreted in terms of direct transitions in the real part of the complex-potential band structure (cf. figure 2). Between 11.0 and $11.5 \mathrm{eV}$, peak $\mathrm{A}$ dominates, with the corresponding final state in the usual real band. From $11.5 \mathrm{eV}$ onwards, this final state moves increasingly into the 


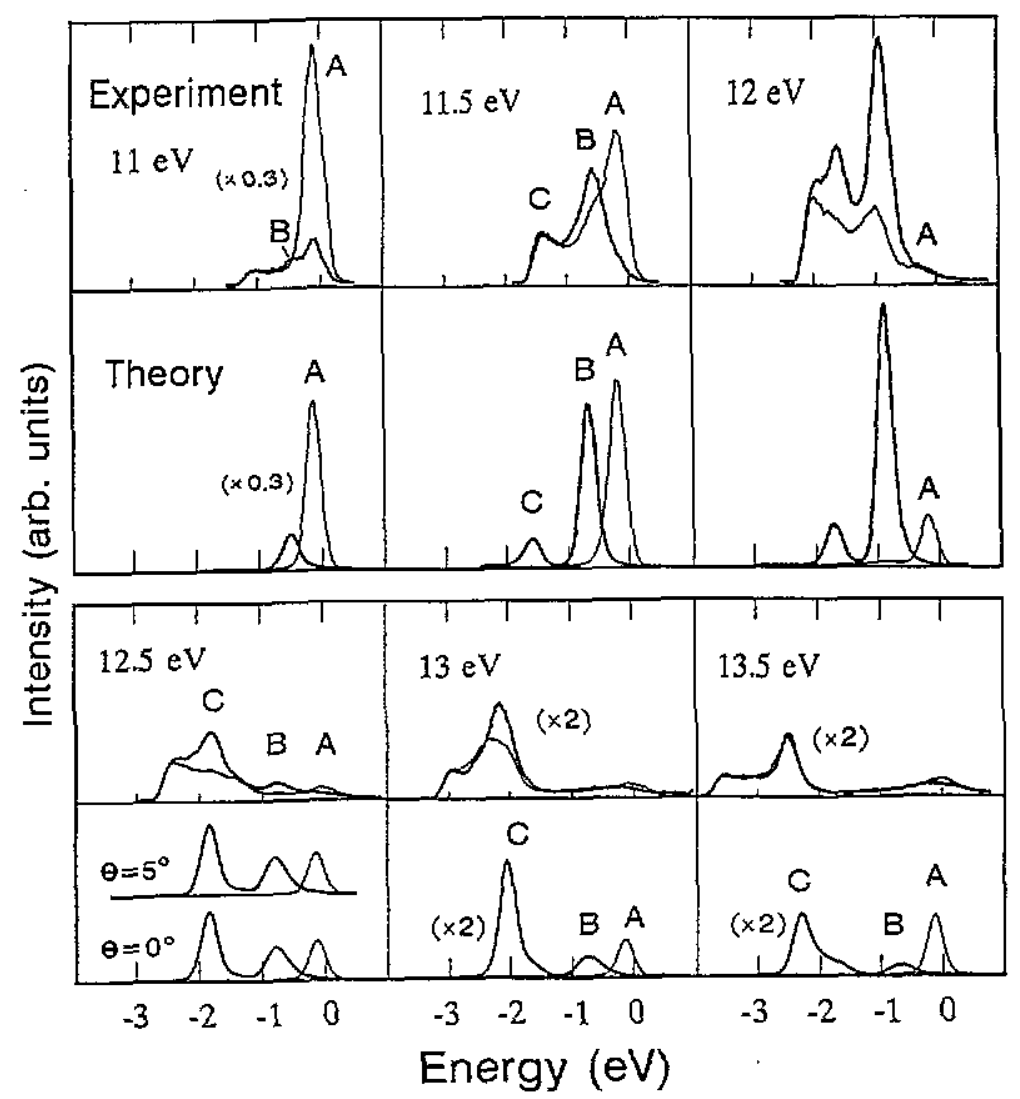

Figure 1. Spin-resolved photoemission intensities $I_{+}$(bold full curves) and $t_{-}$(thin full curves) from $\mathrm{Xe}(111)$ by circularly polarized photons at normal incidence with energies between 11$13.5 \mathrm{eV}$, as indicated. Experiment for normal emission and theory for normal emission and, for $12.5 \mathrm{eV}, \theta=5^{*}$ with azimuthal angle $\phi=0^{\circ}$ relative to miror plane normal to surface. The original measured data points are so closely spaced that they could be replaced by curves. Experimental error bars (not shown) are less than about twice the thickness of the bold curve. The theoretical spectra have been convoluted by a Gaussian of $0.25 \mathrm{eV}$ FWHM. The intensity scales are, separately for experiment and theory, the same for all photon energies unless specified otherwise by a scaling factor in the respective panel (e.g. $(\times 0.3)$ for $11 \mathrm{eV}$ ).

gap, along the dotted 'bridge', with A consequently getting weaker and slightly moving upward in energy. Peak $B$ is weak for $11 \mathrm{eV}$ (although due to a usual direct transition) and subsequently intensifies and disperses downward in accordance with its initial-state band. Between 12.0 and $12.5 \mathrm{eV}$ it collapses due to its final state moving on to the dotted bridge. This behaviour is seen to be in good agreement with experiment. As a point of detail, we note that the down-spin peak $\mathrm{A}$ is also present in the $12 \mathrm{eV}$ experimental data but almost masked in figure 1 by the up-spin spectrum.

'Opposite-spin contributions', which are absent in the calculated curves, are in fact seen to occur in the experimental spectra at all photon energies in figure 1 . One might first think of explaining them in terms of an almost-unpolarized inelastic background. It is, however, striking that the opposite-spin contributions tend to form 'ghost peaks' at the energies of the proper photoemission peaks: see, in particular, at $11 \mathrm{eV}$ under peak $\mathrm{A}$, at 


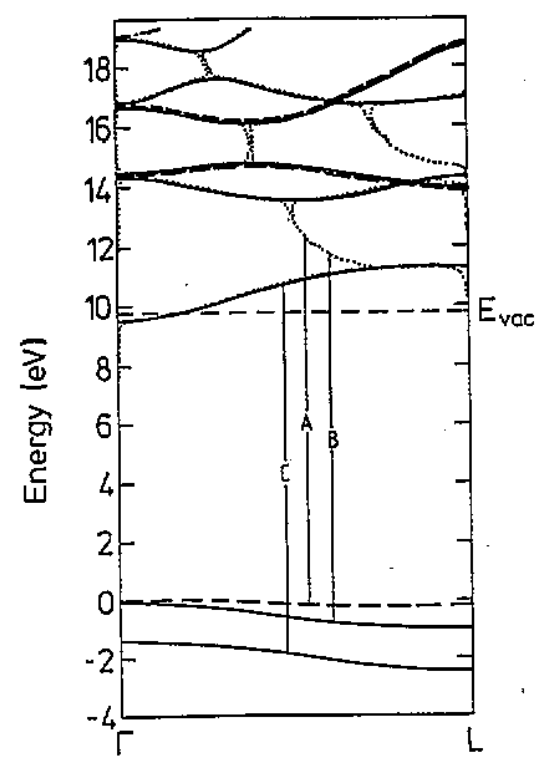

Figure 2. Band structure of Xe along $\Gamma(\Lambda) \mathrm{L}$ : for real potential bands of symmetry types $\Lambda_{6}$ (full curves) and $\Lambda_{4+5}$ (broken curves); above $E_{\text {vac }}$ (horizontal broken line) also real bands for complex potential with $V_{\text {im }}=0.025 \mathrm{eV}$ (dotted curves). Vertical full lines with labels $A, B$ and $C$ indicate direct transitions for the photon energy $12.5 \mathrm{eV}$.

$11.5 \mathrm{eV}$ under $\mathrm{B}$, and from $12.5-13.5 \mathrm{eV}$ under peak $\mathrm{C}$. At $13.5 \mathrm{eV}$, the ghost peak is almost as big as $C$ itself (implying almost zero polarization in contrast to the $+100 \%$ polarization in theory). In search for an explanation we recall that the degree of circular polarization of the incident synchrotron radiation is $(90 \pm 3) \%$ rather than the theoretical $100 \%$. However, this has already been taken into account in the experimental spectra shown in figure 1 (and figure 3). Calculations for slightly off-nomal emission angles (prompted by the finite acceptance angle in experiment) also fail to produce the ghosts. We must therefore look for physical mechanisms, which are present in reality but absent in our theoretical model. We first note that most ghost peaks occur about $1.2 \mathrm{eV}$ above the emission threshold, i.e. are formed by electrons of about $1.2 \mathrm{eV}$ kinetic energy (relative to the vacuum level). From the band structure (figure 2) we see that at this energy there is a flat final-state band (going to the $L$ point). At $13.5 \mathrm{eV}$ one could think of secondary electrons produced by $k$-non-conserving inelastic processes, but this is not possible for the ghost of $\mathrm{A}$ at $11 \mathrm{eV}$, which is quite close to the Fermi energy, i.e. the maximal kinetic energy of photoexcited electrons. What still seems possible, however, are electron-hole scattering processes (due to Coulomb interaction or phonons), which may have a particularly high weight at this energy of very high density of final states. Formally, such processes, which are not spin-selective, can be described by higher-order Keldysh diagrams, in which the two photon vertices are renormalized by electron-hole scattering. We recall that a diagram with one renormalized vertex could actually explain an observed intensity resonance (Gollisch et al 1991). Inclusion of the relevant higher-order diagrams into our photoemission formalism-though rather demanding from the computational point of view-therefore seems a worthwhile undertaking for the future.

Results for higher photon energies, from $14-18 \mathrm{eV}$, are shown in figure 3 . The calculated normal-emission spectra again exhibit the above-discussed peaks A, B and C, which can again be interpreted in terms of the quasi-particle interband transitions, with the appropriate higher final-state energies (cf. figure 2). Comparing with the measured spectra, however, we notice most striking discrepancies. At $14,14.5 \mathrm{eV}$ and $16 \mathrm{eV}$, for example, the sign of the dominant peak is opposite in theory and experiment; at $15 \mathrm{eV}$, the experimental data 


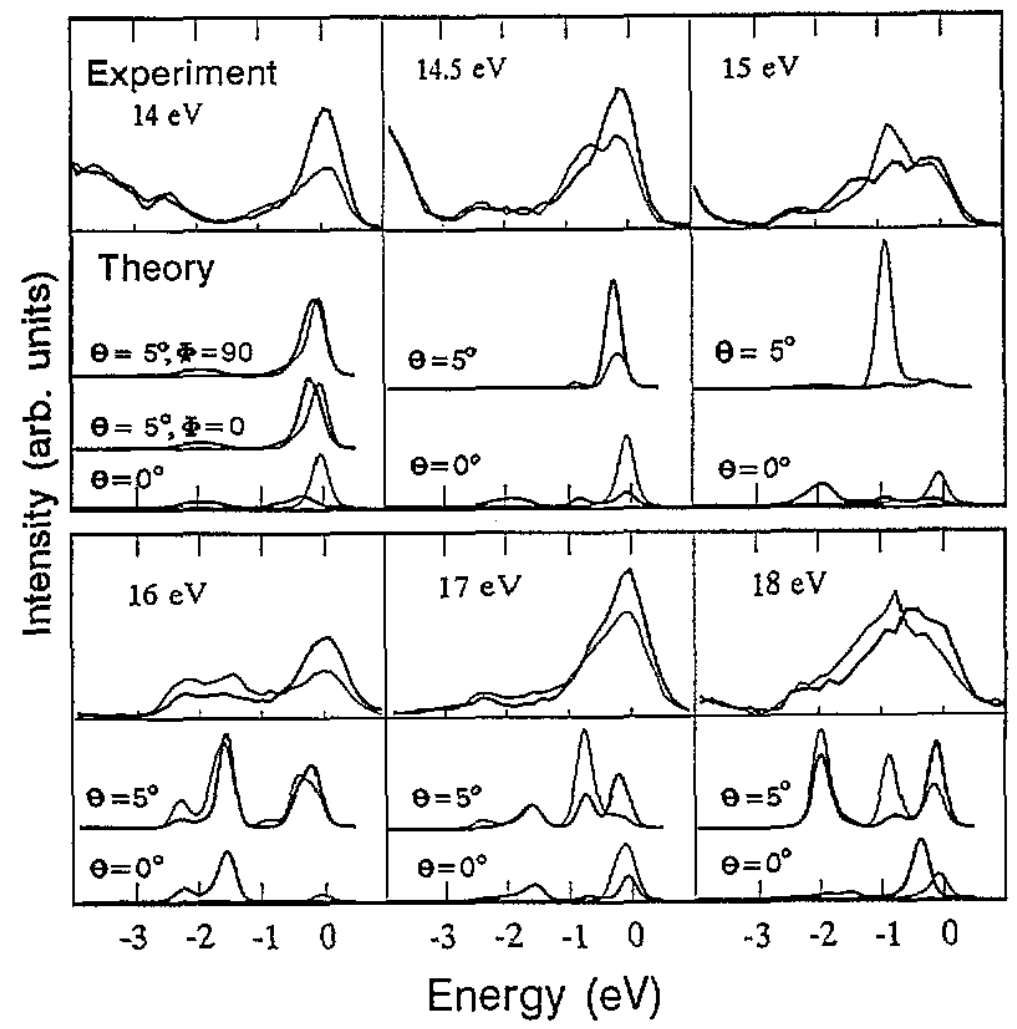

Figure 3. As figure 1 but for photon energies $14-18 \mathrm{eV}$. The measured curves are in normal emission. The calculated curves are for $\theta$ as specified and for $\phi=0^{\circ}$; for $14 \mathrm{eV}$ there is also a $\phi=90^{:}$curve.

exhibit a large $I_{-}$-peak around $-1 \mathrm{eV}$, which is practically absent in the calculated curve. The question arises, then, why the agreement is consistently good for photon energies below $14 \mathrm{eV}$ and just as consistently bad from $14 \mathrm{eV}$ upwards.

In search for an explanation we investigated the effect of the finite emission cone present in the experiment by calculating, for selected photon energies, spectra for $\theta=5^{\circ}$. For photon energies below $14 \mathrm{eV}$, the off-normal emission spectra are almost identical with the normal-emission spectra (cf. the curve for $\theta=5^{\circ}$ in the $12.5 \mathrm{eV}$ panel in figure 1). From $14 \mathrm{eV}$ upwards, however, strong changes occur (see figure 3 ). The off-normal spectra are seen to bring the calculated curves somewhat closer to the experimental data, both with regard to the existence of peaks and the sign of their spin polarization; but strong differences remain. One might now think of reaching better agreement by calculating spectra on a grid covering the experimental emission cone and by summing these spectra with an assumed weight function. However, in view of the uncertainties involved-also with regard to the cone axis relative to the surface normal-we feel that such a fit, even if successful, is not very useful for extracting more physical information, like the refinement involving a slight increase of the final-state self-energy correction with increasing energy.

The origin of the strong sensitivity of the spectra to small changes in the emission angle, from $14 \mathrm{eV}$ upwards, can be traced to the corresponding flat final-state bands (cf. figure 2). Firstly, these bands are found to change more strongly when going to a non-zero 
$k_{\|}$. Secondly, the $\Lambda_{4+5}$ final-state bands cannot couple to the vacuum in normal emission. In the off-normal case, however, hybrids arising from $\Lambda_{4+5}$ and $\Lambda_{6}$ states couple to the vacuum and thus provide additional contributions to the photocurrent via transitions into the $\Lambda_{4+5}$-derived parts.

\section{Conclusion}

Our measured and calculated spin-resolved photoemission spectra agree fairly well with each other for photon energies from 11-13.5 eV, whilst in the photon energy range between $14-18 \mathrm{eV}$ a strong sensitivity to small changes in the emission angle makes it difficult to reproduce experimental data obtained within some finite emission cone. Since the main spectral features in the lower photon energy range can be interpreted in terms of direct transitions in the real part of the complex-potential bulk band structure, the agreement between experiment and theory justifies ex post facto our effective-potential construction and the ensuing quasi-particle band structure. In addition, the experimental data exhibit features of opposite spin ('ghost peaks'), which are absent in the theoretical spectra. We suggest that these features are produced by scattering between the photoelectron and the hole or, in formal terms, by renormalization of the photon vertices in perturbation-theoretical diagrams.

\section{Acknowledgments}

This work was funded by the German Ministry for Research and Technology under contracts $05436 \mathrm{TAB} 6$ and $05331 \mathrm{AX}$. We also gratefully acknowledge the kind hospitality of the Institut für Festkörperforschung of the Forschungszentrum Jülich and of BESSY, Berlin.

\section{References}

Ackermann B 1985 PhD Thesis Universität Duisburg

Ackermann B and Feder R 1985a J. Phys. C: Solid State Phys. 181093

- 1985b Solid State Commun. 541077

Bacalis N C, Papaconstantopoulos D A and Pickett W E 1988 Phys. Rev. B 386218

Eyers A, Schäfers F, Schönhense G, Heinzmann U, Oepen H P, Hünlich K, Kirschner J and Borstel G 1984 Phys. Rev. Lett. 521559

Feder R 1985 Polarized Electrons in Surface Physics ed R Feder (Singapore: World Scientific)

Gollisch H, Meinert D, Tamura E and Feder R 1991 Solid State Commun. 82197

Heinzmann U 1990 Photoemission and Absorption Spectroscopy of Solids and Interfaces with Synchrotron Radiation ed M Campagna and R Rosei (Amsterdam: North-Holland)

Heinzmann U and Schönhense G 1985 Polarized Electrons in Surface Physics ed R Feder (Singapore: World Scientific)

Kessler B, Eyers A, Horn K, Müller N, Schmiedeskamp B, Schönhense G and Heinzmann U 1987 Phys. Rev. Lett. 59331

Kirschner J 1985 Polarized Electrons at Surfaces (Springer Tracts in Modern Physics 106) (Berlin: Springer)

Kittel C 1971 Introduction to Solid State Physics 4th edn (New York: Wiley) p 38

Meier F 1985 Polarized Electrons in Surface Physics ed R Feder (Singapore: World Scientific)

Pendry J B 1976 Surf. Sci. 57679

Schäfers F, Peatman W, Eyers A, Heckenkamp Ch, Schönhense G and Heinzmann U 1986 Rev. Sci. Instrum. 57 1032

Schneider C M, Garbe J, Bethke K and Kirschner J 1989 Phys. Rev. B 391031 
Stoppmanns P, Heidemann B, Irmer N, Muller N, Vogt B, Schmiedeskamp B, Heinzmann U, Tamura E and Feder R 1991 Phys. Rev. Lett. 662645

Tamura E and Feder R 199ta Solid State Commun. 79989

- 1991b Europhys. Lett. 16695

Tarnura E, Feder R, Vogt B. Schmiedeskamp B and Heinzmann U 1989 Z. Phys. B 77129

Tamura E, Piepke W and Feder R 1987 Phys. Rev. Lett. 59934

von Barth U and Hedin L 1972 J. Phys. C: Solid State Phys. 51629

Woehlecke M and Borstel G 1984 Optical Orientation (Modern Problems in Condensed Matter Sciences 8) ed F Meier and B P Zakharchenya (Amsterdam: North-Holland)

Wyckoff R W G 1982 Crystal Structures (Malibar, FL: Krieger) p 4 